\title{
Cómo desarrollar una plataforma de formación a distancia para el entorno industrial. $P Q P$ «Plataforma para la prevención de Riesgos Laborales»
}

\author{
(How to develop a distance education platform for an industrial \\ environment. \\ $P Q P$ «Platform for the prevention of occupational hazards») \\ NURIA LLORET ROMERO \\ Universidad Politécnica de Valencia \\ (España) \\ Margarita Cabrera MÉNDEZ \\ Mas Medios S.L. \\ (Valencia, España)
}

\footnotetext{
RESUMEN: La Plataforma para la Calidad en la Prevención es un proyecto dentro de la iniciativa europea $A D A P T$ que desarrolla, en Internet, una herramienta útil para la búsqueda de soluciones en la Prevención de Riesgos Laborales, la Red PQP. Su finalidad es facilitar a los usuarios la obtención de forma directa y cómoda de información, comunicación, formación y asesoramiento sobre la Prevención de Riesgos Laborales. PQP incorpora las ventajas de las nuevas tecnologías de la información y comunicación en un entorno web al mundo de la Prevención. El curso básico en prevencion de riesgos laborales y su metodología de actuación, partiendo de una concepción de cooperación, suma de esfuerzos y recursos, al servicio de la acción preventiva, permite:

- Un Asesoramiento personalizado

- Una Formación adecuada a las necesidades de cada usuario

- Una Gestión de la Prevención guiada
} 
- Una canalización de todos los recursos de información para avanzar en una gestión de la prevención basada en la calidad.

De manera que al integrarse en la nueva cultura tecnológica, se sirven de las ventajas que esta ofrece:

- La posibilidad de elegir cuándo y dónde estudiar

- Una formación personalizada y adaptada al alumnado

- Una efectiva participación e interactividad del alumno

- Unos contenidos siempre actualizados y de fácil manejo

- El apoyo y la comunicación continua de tutores especialistas

- El acceso a recursos telemáticos actualizados periódicamente

Está basado en un aprendizaje activo, práctico y dinámico. Se requiere de una alta participación por parte del alumno en su proceso de formación. Apoyado en todo momento de recursos telemáticos que guiarán su aprendizaje y de un equipo de tutores para resolver cualquier duda planteada.

Curso basado en la web - sistemas y herramientas - e-learning - multimedia en software educativo

ABSTRACT: The Platform for Quality in Prevention is a project within the European initiative ADAPT, with the aim to develop a Web-based tool to be used to find solutions in Prevention of Occupational Hazards, called the PQP Network. It tries to help users to find sources of information, communication, training and advice on prevention of occupational hazards in a direct and convenient way.

PQP takes the benefits of working with the new Internet information and communication technologies to the prevention field. The basic course on Prevention of Occupational Hazards, which focuses on cooperative work and putting together efforts and resources to improve prevention methods, allows:

- Individual assessment

- Individual training to meet different user's needs

- Guided prevention management

- Channelling of all the information resources available to achieve prevention management focused on quality.

Working in this new technologies environment means more benefits, such as:

- Training sessions can take place any time, anywhere

- Individual training that meets the user's needs

- The user actively participates in the sessions

- Contents are constantly updated and are easy to use

- Tutors are always available to support the user

- Access to regularly updated Internet resources

The main concept is active, practical and dynamic learning. A high degree of participation in the training process is required from the user. They will be supported at all times by Internet resources to guide their learning, and by a team of tutors who will deal with any query they may have.

Web-based courseware - systems and tools - E-learning - Multimedia in educational software.

\section{INTRODUCCIóN}

El nuevo marco normativo sobre Prevención de Riesgos Laborales (31/1995 de 8 de noviembre) supone un profundo cambio en las empresas, incorporar la necesidad de la gestión preventiva. La necesidad de dar cumplimiento a la LPRL, que entró en vigor el 10 de febrero de 1996, tiene por objeto la determinación de garantías, y responsabilidades necesarias para desarrollar un nivel adecuado de protección de la salud de los trabajadores frente a los riesgos derivados de las condiciones de trabajo. A pesar de la obligatoriedad del cumplimiento de la normativa el nivel de siniestralidad por accidentes de trabajo sigue creciendo en todos los países. 
Este nuevo entorno precisa el desarrollo de una organización preventiva. Un entorno capaz de dar respuesta a las necesidades de gestión de los riesgos laborales y de alcanzar la integración de la prevención en todas y cada una de las actividades desarrolladas por los trabajadores en la empresa.

La RED PQP se configura como una herramienta útil, ágil y rentable para el cumplimiento de la nueva normativa. Es capaz de dar respuesta a las características de cada empresa en función de su actividad, salvando las distancias de espacio y tiempo. El asesoramiento por expertos, la formación continua en prevención, los foros de discusión, la amplia base documental en prevención, entre otros servicios, unidos a Internet como fuente de información, contribuyen a su éxito.

La Red PQP, Plataforma para la Calidad en la Prevención, pretende ser un puente entre el usuario y la Prevención, apoyándose en las nuevas tecnologías de la información, una herramienta útil para la gestión preventiva.

Los usuarios, pueden acceder de modo gratuito a la condición de miembros de la red. El estatus de miembro proporciona una serie de ventajas especiales como: disponer de un servicio de asesoramiento, integrarse en seminarios de formación para miembros, acceso a publicaciones en red, becas para miembros, preferencia en acciones de formación con plazas limitadas, bonificaciones en matrículas, una cuenta de correo propia y otros que irán definiéndose en un futuro.

Redpqp.com, «Plataforma: Calidad y Prevención», [URL: http://www.red pqp.com] es un proyecto basado en la cooperación. Tiene como objetivo principal contribuir a crear un entorno de trabajo seguro y saludable, dirigido a proporcionar las condiciones óptimas para alcanzar mayores cotas de productividad, eficiencia, calidad y competitividad en el trabajo.

En diciembre de dos mil la redpqp.com cierra una primera etapa con un bagaje muy positivo, que ha sido posible gracias al proyecto desarrollado en el marco de la Iniciativa Europea ADAPT, destinada a aportar propuestas que permitan adaptar los recursos humanos a las nuevas necesidades planteadas por las pymes.

\section{OBJETIVOS}

Siguiendo el hilo de las acciones destinadas a la mejora de la calidad de la enseñanza y la adecuación de los usuarios al nuevo entorno del e-learning, se han establecido los objetivos según el impacto en los siguientes aspectos:

\section{A) Pedagógicos}

D Ofrecer al alumno todos los recursos integrados de información para cada asignatura y para todo el área de conocimiento desde una misma interface. 
D Con el Curso Básico de Prevención de Riesgos Laborales se pretende establecer la formación básica que facilite las actitudes y aptitudes para la acción preventiva en las empresas.

D Fomentar la participación activa en la prevención y protección de la seguridad y salud en el trabajo.

D Conceder la posibilidad al alumno de poder elegir cuándo y dónde estudiar.

D Una efectiva participación e interactividad del alumno, logrando su implicación en la materia mediante fórmulas intuitivas.

D Contenidos siempre actualizados y de fácil manejo.

D El apoyo y la comunicación continua de tutores especialistas.

D Facilitar al alumno un sistema que aglutine herramientas de apoyo en su formación en prevención.

D El acceso a recursos telemáticos actualizados periódicamente.

B) Docentes

D Conseguir que el usuario a través de situaciones semi reales pueda evaluar o prevenir los riesgos sin necesidad de que el alumno tenga que vivir la situación real.

D Fomentar la integración del equipo de profesores, así como de especialistas en prevención de riesgos y alumnado en un proyecto común.

D Formar a las empresas en la prevención de riesgos.

D Eliminar barreras geográficas y horarias.

C) Tecnológicos

D Poner en marcha el servidor web que permita la utilización de los recursos existentes en la red PQP para la asimilación por parte de los usuarios de los conceptos básicos en prevención de riesgos laborales.

D Incluir y actualizar cada vez mayor número de recursos relacionados con la prevención: bibliotecas, videotecas, hemerotecas, enlaces, legislación, bases de datos de primeros auxilios...

D Integrar herramientas basadas en las nuevas tecnologías: biblioteca virtual, foros, bolsa de trabajo, cursos... en el entorno de la prevención.

\section{METODOLOGÍA}

Su metodología se basa en la consecución de los objetivos formativos por la interacción profesor alumno y entre alumnos a través de medios telemáticos. 
Cómo desarrollar una plataforma de formación a distancia para el entorno industrial. PQP «Plataforma...»

Asíncrona cuando no es necesario que la interactividad entre las personas se produzca simultáneamente.

Es muy característico de las aulas virtuales que tengan una serie de servicios para el apoyo a la formación, tales como:

D Asesoramiento personalizado mediante un tutor en línea

D Documentación que incluye biblioteca y hemeroteca digital, legislación, enlaces, documentos para bajarse desde la red...

D Servicio de foros y chats

D Apartado de novedades, noticias, agenda, tablón...

D Una cafetería, que suele ser un servicio de news o un chat para que los alumnos se puedan relacionar.

D Formularios de envío de datos

Dona de exámenes...

La Red PQP está basada en la integración de este tipo de herramientas vía internet que faciliten a los usuarios el aprendizaje de la prevención de riesgos, uniendo la formación clásica con los distintos elementos auxiliares que proporcionan las nuevas tecnologías. Para todo ello se creó el aula virtual, que como su nombre indica pretende ser una copia de un aula o escuela vía web.

Se le dotó de los diferentes elementos que integran un aula: los contenidos, que en este caso se corresponden con los cursos. Los materiales auxiliares de for-

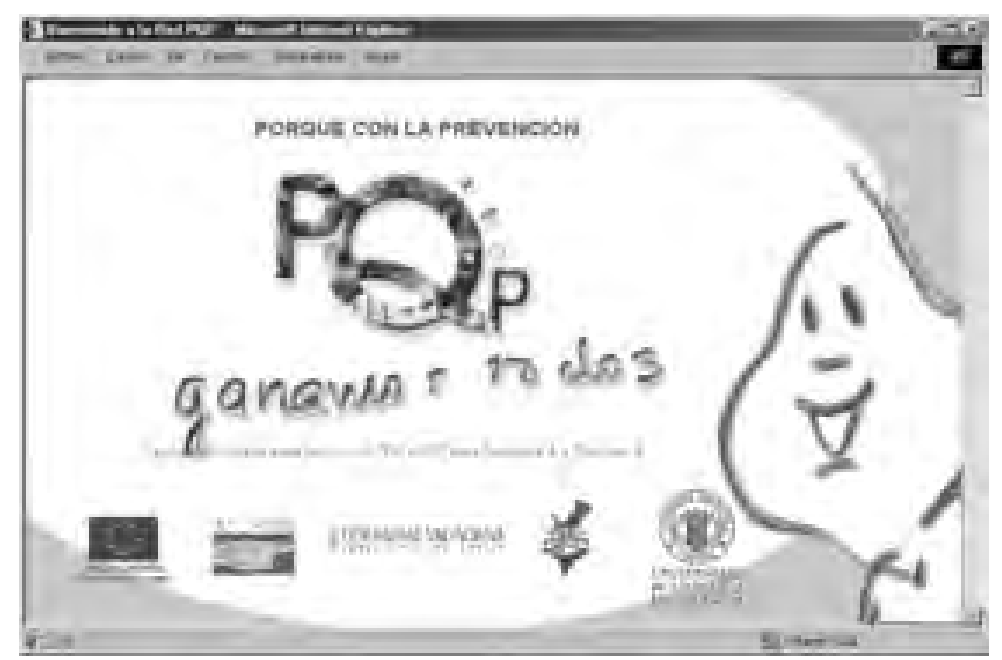

Figura 1. Pantalla de entrada. 
mación, que se encuentran integrados en la zona de documentación. Una zona de gestión de la prevención que equivale a lo básico que el alumno debe conocer. El espacio de los profesores o especialistas donde poder preguntar y resolver dudas: asesoramiento. El área de compartir con profesores y alumnos, así como con especialistas: foros, tanto formales como informales. Y por última un espacio reservado para novedades, donde tiene cabida el tablón de anuncios y la agenda.

Se hizo un interface al estilo de las aulas convencionales, con un tablero verde y formas sinuosas que invitan a conformar un ambiente relajante y de estudio. Del mismo modo se hizo especial hincapié en lograr unas imágenes amenas y al mismo tiempo sencillas de manera que el usuario identificase la facilidad del aprendizaje y no molestara a la hora de leer a través de la pantalla. Cabe destacar la dificultad del proyecto en el sentido de que debía ser desarrollado para todo tipo de usuarios, esto es, desde la persona que se encuentra a cargo de una máquina de una fábrica, hasta el directivo o el empresario. Es por ello que tanto el diseño como la navegación son muy sencillas de manejo y complejidad, creando un ambiente sereno y de apariencia relajante.

Los contenidos están estructurados en la pantalla según importancia. En la parte superior encontramos la navegación principal, entendemos por navegación principal los enlaces desde los que se puede acceder a las grandes áreas temáticas: formación, documentación, gestión de la prevención, asesoramiento, foros y novedades.

En la zona inferior de todas las pantallas encontramos una navegación auxiliar que nos ayuda a movernos por todo el aula. Ésta se compone de los siguientes elementos: búsqueda, mapa del web y bolsa de empleo. Así como preguntas más frecuentes sobre el aula en general y sobre cada apartado.

Vamos a analizar a continuación las diferentes áreas que integran el aula virtual:

\section{Documentación}

La zona de documentación pretende integrar los diferentes formatos de información que son significativos para la gestión de la prevención. Encontramos en el segundo nivel las siguientes opciones:

- Enlaces. Se han incluido enlaces a organismos públicos y privados a nivel nacional e internacional, asociaciones y mutuas. Además se listan todas las referencias a la legislación de interés para la prevención. Enlaces a normalización, Instituciones y buscadores de internet.

- Biblioteca. Legislación de Referencia, Artículos Científicos, Publicaciones Periódicas, Monografías, Bases de datos de recursos en Internet. Todo ello apoyado por un buscador interno que facilite la consulta. 


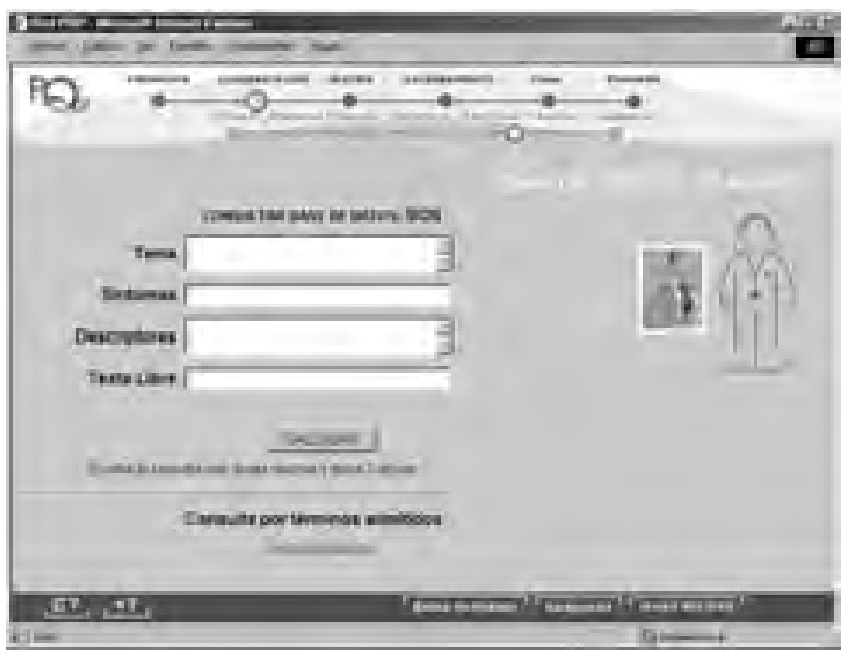

Figura 2. Pantalla de consulta de primeros auxilios.

- Hemeroteca y Videoteca. Zona de referencia donde se puede buscar información en formato revista, prensa o video relacionada con la prevención.

- Bases de datos de primeros auxilios. Zona de consulta rápida sobre primeros auxilios donde se puede buscar por tema, síntoma, descriptor o testo libre.

- Bases de datos de legislación. Zona de consulta rápida sobre legislación relacionada donde se puede buscar por tema, síntoma, descriptor o testo libre. Tanto estas bases de datos como las de primeros auxilios se actualizan periódicamente de manera que el usuario siempre pueda estar informado.

\section{Gestión de la prevención}

La gestión de la prevención debe integrarse en el organigrama general de la empresa y debe impregnar y actuar en todas las áreas, departamentos, estamentos y personas que compongan la organización.

En este contexto, los conceptos de prevención y calidad deben ir estrechamente unidos, puesto que con la prevención intentamos conseguir un gran objetivo: la consecución de calidad en las condiciones de vida y trabajo de los trabajadores.

Todo esto es lo que se define y explica en esta zona del aula: política de prevención, organización de la prevención en la empresa, y documentación de las actividades preventivas. 


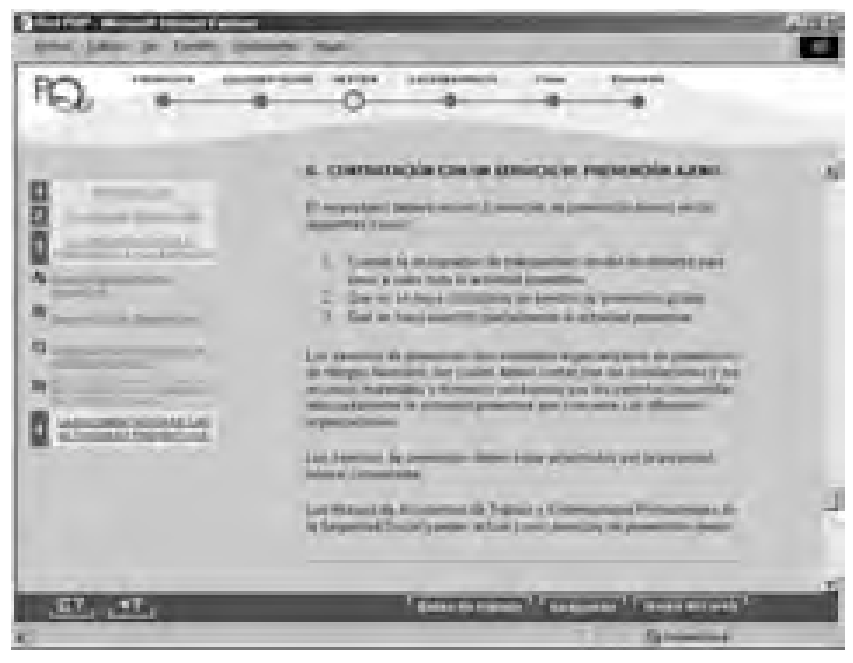

Figura 3. Pantalla de gestión de la prevención.

\section{Asesoramiento}

El área de asesoramiento se ha dividido a su vez en dos zonas: en primer lugar una que podríamos llamar pública donde podemos mostrar a través de un tablón nuestras preguntas o inquietudes relacionadas con la prevención. Una vez publicadas puede responderlas tanto el profesor, como otro alumno, como uno de los especialistas de la red PQP.

En la zona privada hacemos la pregunta directamente al profesor o especialista y nos contesta directamente sin necesidad de que el resto de la comunidad lo lea.

\section{Foros}

Servicio abierto continuamente en el que, mediante el correo electrónico, el usuario dispone de un equipo de especialistas que darán asesoramiento a tiempo real. Se ha dividido en dos zonas: pasillo, que es un sistema de conversación a tiempo real donde el usuario interviene en una conversación múltiple desde su ordenador a modo de discusión interactiva sobre un tema concreto en el que pueden aportar sus conocimientos, dudas, o particular punto de vista.

Igual que «pasillo», «espiral» es una lista de distribución voluntaria, pero se activa en un periodo muy corto de tiempo: se trata de un debate serio sobre un tema específico con alguien que sabe mucho del tema. 


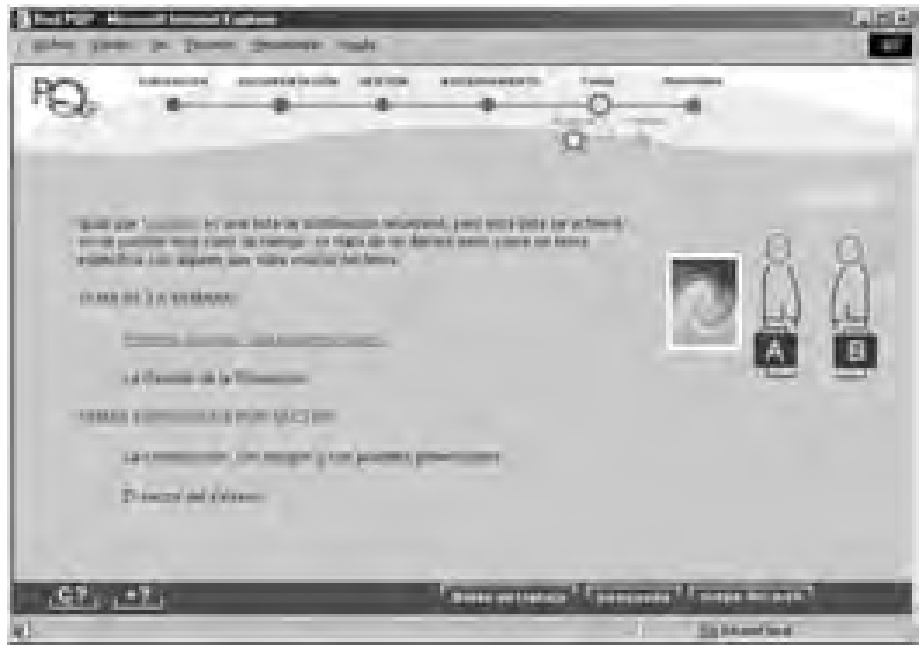

Figura 4. Pantalla de espiral.

\section{Novedades}

En un contexto de aprendizaje autónomo, es muy importante el estar al día. Para ello se ha creado la zona de novedades. Dentro de novedades hemos incluido la agenda, una base de datos de prensa actualizada en todos los temas de prevención y un tablón de anuncios donde tanto los profesores, como los alumnos y

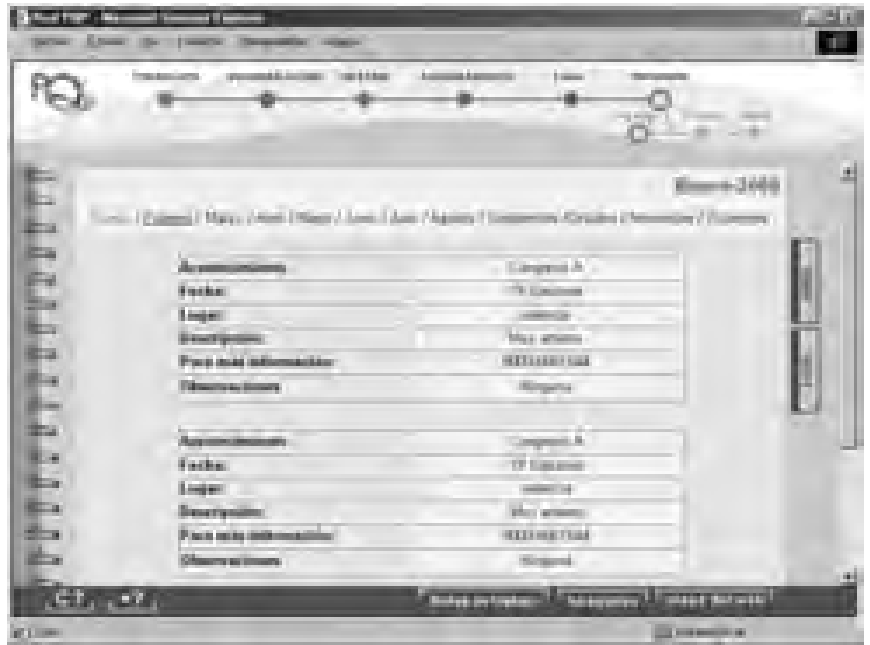

Figura 5. Pantalla de agenda. 


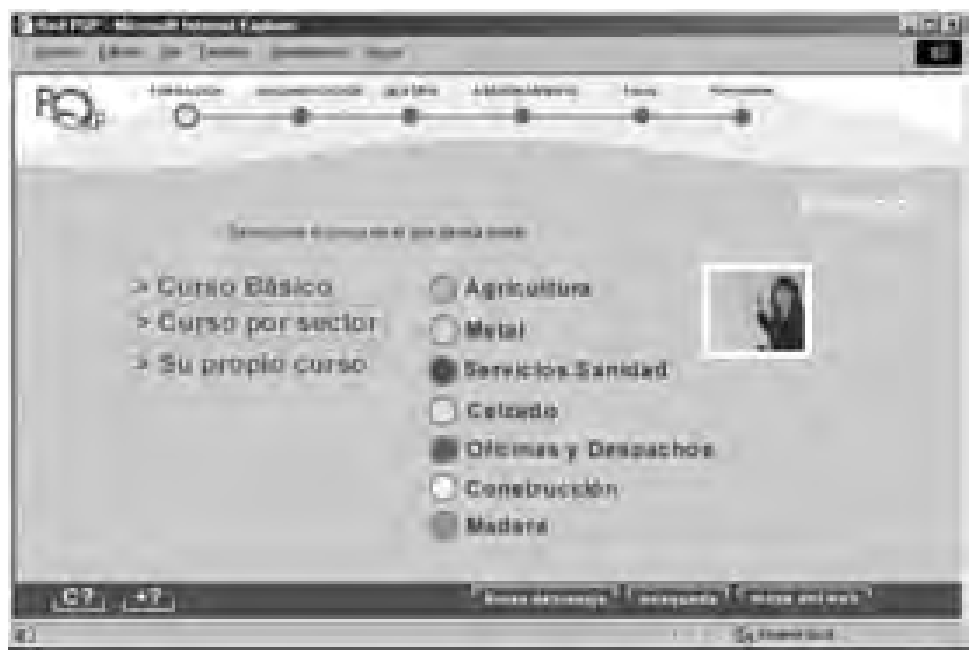

Figura 6. Pantalla de formación.

especialistas pueden publicar todo lo que les interesa relacionado con el mundo de la prevención.

\section{Formación}

El proyecto presentado, totalmente innovador en la línea de los programas de formación en prevención de riesgos laborales, contiene en el aula distintos cursos según el sector o el nivel. Se ha diferenciado en primer lugar un curso básico donde se tratan todos los temas comunes a todos los sectores y niveles.

A continuación se han realizado cursos por sectores donde se abarca la prevención específica de cada sector. Y por último se ha dejado un área abierta donde las distintas empresas pueden solicitar a la empresa.

\section{Curso básico}

Los contenidos están estructurados en la pantalla según importancia. En la parte superior encontramos la navegación principal (Se entiende por navegación principal los enlaces desde los que se puede acceder a los diferentes temas de los cursos) y los siguientes módulos siguiendo la estética del aula virtual:

\section{1) MÓDULO I Marco Normativo}

1. Introducción.

2. Ley de prevención de riesgos laborales. 


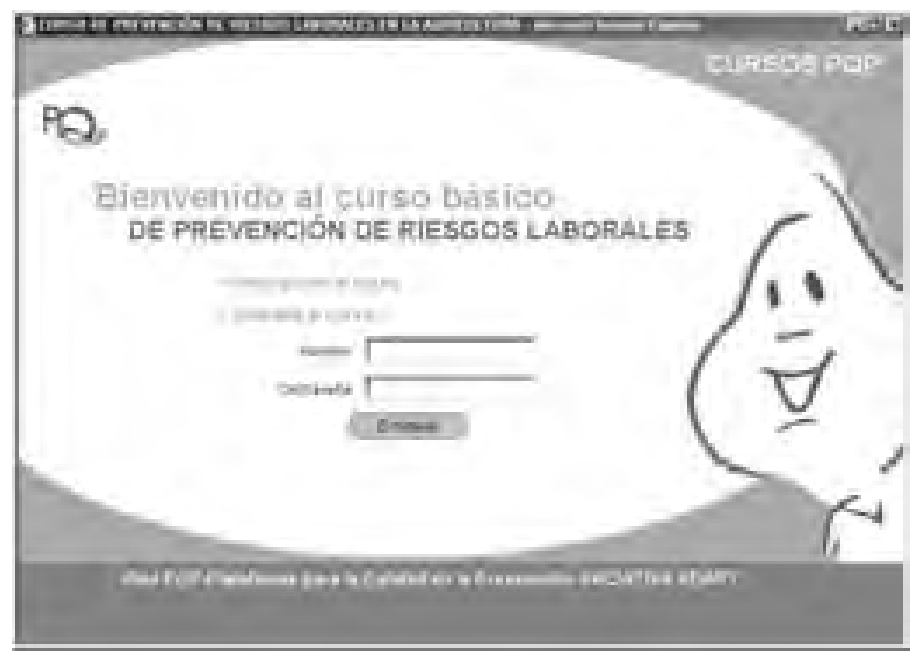

Figura 7. Pantalla de entrada al curso básico.

3. Política en materia de prevención de Riesgos Laborales.

4. Normas reglamentarias.

5. Derechos y obligaciones.

6. Representación y participación de los trabajadores.

2) MÓDULO II Condiciones de Seguridad y Salud

1. Conceptos Básicos.

2. Condiciones de Seguridad: Seguridad en el Trabajo y Planes de emergencia.

3. Los riesgos ligados al medio ambiente en el trabajo.

4. Las características y la organización del trabajo.

5. El control de la salud de los trabajadores.

6. Primeros auxilios.

3) MODULO III Gestión de la prevención

1. Introducción.

2. Política de prevención de riesgos laborales.

3. La organización de la prevención en la empresa.

4. La evaluación de Riesgos Laborales.

5. El plan de prevención de riesgos laborales. 


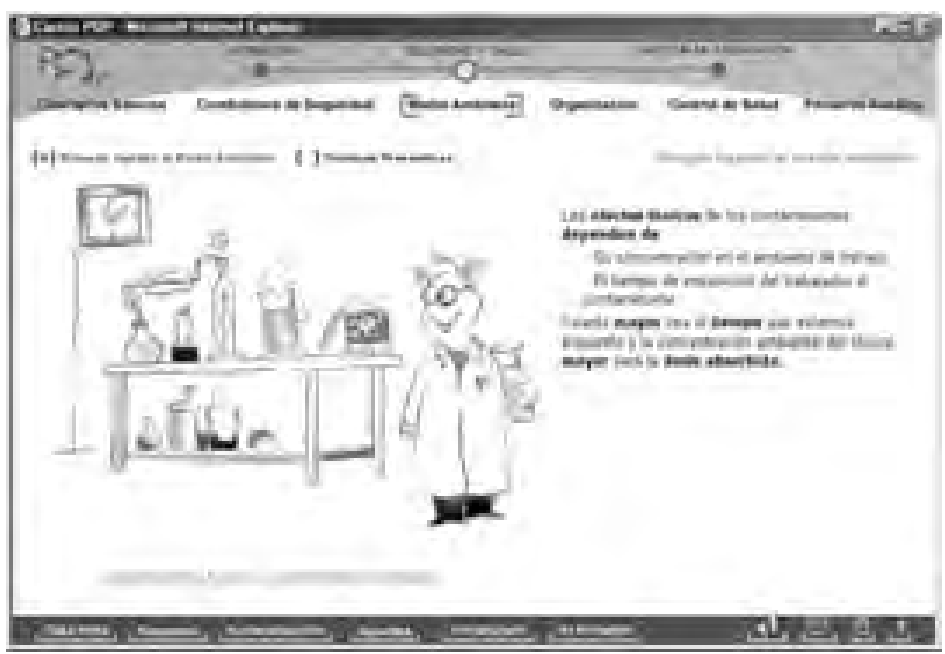

Figura 8. Pantalla interior de un curso.

6. La documentación de las actuaciones preventivas.

7. El control de la prevención.

8. Navegación auxiliar específica

Se entiende por navegación auxiliar específica los enlaces desde los que se puede acceder a los diferentes recursos que tiene cada página, o módulo, donde el alumno puede localizar las distintas ayudas relacionadas con cada tema en particular para apoyar su formación:

- Apuntes

- Vocabulario

- Resumen

- Autoevaluación

- Actividades

- Guía tema

\section{Navegación auxiliar}

Se entiende por navegación auxiliar el enlace desde el que se puede acceder a los diferentes recursos pedagógicos que tiene el curso en general.

- Guía didáctica

- Tutoría 
- Índice

- Ayuda

\section{CONCLUSIONES}

- El proyecto permite aglutinar a nivel nacional gran cantidad de información para todos los implicados en la gestión de la prevención anulando barreras geográficas y temporales.

- El aula virtual ha permitido que el alumno tenga un contacto directo con los especialistas, profesores y personas implicadas en la prevención de riesgos laborales mediante un foro de encuentro.

- El sistema permite que la actualización de datos sea continua y mantenga al alumno informado de las últimos requisitos sobre riesgos laborales dando información de primera mano.

- El proyecto ha conseguido que un tema legal y de normativa difícil de digerir para el alumno sea amigable y permita un aprendizaje práctico e intuitivo con casos aplicados.

- La interactividad. El alumno llega a ocupar el papel activo en el proceso de comunicación, por lo que aporta la bidireccionalidad de la red y se adquiere la responsabilidad de ser al mismo tiempo emisor y receptor.

- Se aumentan las posibilidades de la enseñanza permanente ya que el estudiante puede seguir formándose adquiriendo no sólo conocimientos sino la destreza para utilizar las nuevas tecnologías en el acceso a la información y a la formación independientemente de su situación laboral o residencia real.

- Los formadores tienen los conocimientos técnicos suficientes para utilizar con mayor optimización los recursos de las nuevas tecnologías.

- Adecuación de temarios al soporte electrónico realizando análisis metodológicos y de interface de usuario. 


\section{REFERENCIAS BIBLIOGRÁFICAS}

Abascal, Julio, J.J. Cañas, M. Gea, J. Lores, M. Ortega, L.A. Ureña y M. Vélez. Curso Virtual de Introducción a la Interacción Persona-Ordenador. http://griho.udl.es/ipo.

Ainsworth D. (1987). What century is this anyway? A critical look at technology in education. Educ Technol.

Bou BauZÁ, G. (1997). El guión multimedia. Anaya multimedia. Madrid 1997.

Dinucci D., Giudice M. y Stiles L. (1998). Elements of web design. Peachpit Press. USA.

DonNelly D. (1997) Web pages from around the world. Rockport Publishers. USA.

Ferrés, J. y MarquÉs, P. (1996) Comunicación educativa y Nuevas Tecnologías. Praxis. Barcelona.

García Valcárcel, A. Y Tejedor, Fco. J.(1996) Perspectivas de las nuevas tecnologías en educación. Narcea. Madrid.

González Castro, V. (1986). Teoría y práctica de los medios de enseñanza. Editorial Pueblo y Educación. La Habana.

HeID, J. (1998). Los secretos de HTML y creación de páginas web a tu alcance. Anaya Multimedia. Madrid.

Kent G. (1969). Blakboard to computer. Ward Lock Education. Londres.

Kristof, R. y Satran A. (1998) Diseño Interactivo. Anaya Multimedia. Madrid.

LóPEz Ostio J. (1993) Sistemas Tutoriales Inteligentes (ITS). Conferencia mecanografiada. San Sebastián.

Mandel, Theo. (1997). The elements of user interface design. Wiley Computer Publishing.

Monguet Fierro, J. M. y Fernández SÁnchez, J. Escenarios a corto y medio plazo para la formación continua a distancia. http://www.ucm.es/info/multidoc/multidoc/revista/num8/ monguet.html.

Moreno Muñoz, A. (1999). Diseño ergonómico de aplicaciones hipermedia. Paidós. Barcelona.

Mullet K. y SANo D. (1995). Designing visual interfaces. Sun Microsystems, Inc. USA.

O’SHEA T, SELF J. (1989) Enseñanza y aprendizaje con ordenadores. Editorial Científico-Técnica. La Habana.

Pablos J., Jiménez J. (1998) Nuevas Tecnologías, Comunicación audiovisual y Educación. Cedecs. Barcelona.

PÉrez Alarcón, A., Serrano Muñoz, J. Tecnologías de la información aplicadas a los servicios bibliotecarios. http://www.ucm.es/info/multidoc/multidoc/revista/num8/bib-vir.html.

Raskin, J. (2000). The humane interface. Addison Wesley.

Rossell W. (1989). Medios de enseñanza. Editorial Pueblo y Educación. La Habana.

ROVIRA, C. La orientación a objetos en el diseño de hipertextos para la enseñanza - aprendizaje. http://www.ucm.es/info/multidoc/multidoc/revista/num8/siguenza.html.

SAETLER P. (1968). A history of instructional technology. Mc Graw Hill Books. USA.

SIEGEL D. (1997). Técnicas avanzadas para el diseño de páginas web. Anaya multimedia. Madrid.

SIGÜENZA, J. A. Diseño de materiales docentes multimedia en entornos virtuales de enseñanzaaprendizaje. http://www.ucm.es/info/multidoc/multidoc/revista/num8/siguenza.html. 
Cómo desarrollar una plataforma de formación a distancia para el entorno industrial. PQP «Plataforma...»

Swann A. (1993) Cómo diseñar retículas. Manuales de diseño, Gustavo Gili. Barcelona.

WiLDBUR P. y BURKE M. (1998). Infográfica, soluciones innovadoras en el diseño contemporáneo.

Gustavo Gili. Barcelona.

\section{PERFIL ACADÉMICO Y PROFESIONAL DE LAS AUTORAS}

Nuria Lloret Romero es subdirectora de proyectos de I+D en la Universidad Politécnica de Valencia, dentro del Departamento DCADHA. Asimismo es socia fundadora consultora en proyectos para la empresa MAS MEDIOS y participa como experta para la evaluación de proyectos de la Unión Europea para el Programa de Sociedad de la Información.

Nuria Lloret Romero

Universidad Politécnica de Valencia

Camino de la Vera, s/n

42061 Valencia (España)

correo electrónico: NLLORET@UPVNET.UPV.ES

Margarita Cabrera Méndez es socia fundadora de Mas Medios par la Gestión de la Información S.L., empresa en la que desarrolla su labor profesional como gerente y responsable del departamento de contenidos.

Margarita Cabrera Méndez

C/ Álvaro de Bazán, 10

46010 Valencia (España)

correo electrónico: mcabrera@masmedios.com 